\title{
Letermovir for the prevention of cytomegalovirus infection and disease in transplant recipients: an evidence-based review
}

This article was published in the following Dove Press journal:

Infection and Drug Resistance

\author{
Guy El Helou ${ }^{1,2}$ \\ Raymund R Razonable ${ }^{1,2}$ \\ 'Division of Infectious Diseases, \\ Department of Medicine, Mayo Clinic, \\ Rochester, MN, USA; ${ }^{2}$ William J von \\ Liebig Center for Transplantation and \\ Clinical Regeneration, Mayo Clinic, \\ Rochester, MN, USA
}

\begin{abstract}
Cytomegalovirus (CMV) is a leading opportunistic infection in immune compromised patients, including allogeneic hematopoietic stem cell (HSCT) or solid organ transplant (SOT) recipients, where primary infection or reactivation is associated with increased morbidity and mortality. Antiviral drugs are the mainstay for the prevention of CMV infection and disease, most commonly with valganciclovir. However, valganciclovir use is often associated with adverse drug reactions, most notably leukopenia and neutropenia, and its widespread use has led to emergence of antiviral resistance. Foscarnet and cidofovir, however, are associated with nephrotoxicity. Letermovir, a novel CMV viral terminase inhibitor drug, was recently approved for CMV prophylaxis in allogeneic HSCT recipients. It has a favorable pharmacokinetic and tolerability profile. The aim of this paper is to review the evidence supporting the use of letermovir in allogeneic HSCT recipients, and how the drug impacts our contemporary clinical practice. In addition, we discuss the ongoing clinical trial of letermovir for the prevention of CMV in SOT recipients. The use of letermovir for treatment of CMV infection and disease is not yet approved. However, because of a unique mechanism of activity, we provide our perspective on the potential role of letermovir in the treatment of ganciclovir-resistant CMV infection and disease. Furthermore, drug-resistant CMV has emerged during use of letermovir for prophylaxis and treatment. Caution is advised on its use in order to preserve its therapeutic lifespan.
\end{abstract}

Keywords: letermovir, valganciclovir, cytomegalovirus, UL56, UL97, transplantation

\section{Introduction}

Cytomegalovirus (CMV) is a major cause of morbidity in immunocompromised hosts, including allogeneic hematopoietic stem cell transplant (HSCT) or solid organ transplant (SOT) recipients. In healthy individuals, primary infection with CMV is often asymptomatic, or it manifests with a self-limited febrile illness. After primary infection, CMV goes into a state of latency, with occasional episodes of viral reactivation. Innate and adaptive immune responses provide adequate control of CMV infection, with the former acting during the initial stages of primary infection and the latter playing a major role at later stages, notably with the development of CMV-specific CD4 and CD8 T cells ${ }^{1,2}$ and CMV-specific antibodies.

\section{Risk factors of CMV in transplantation}

There are many factors that increase CMV infection risk and the extent of disease after transplantation (Table 1). Among SOT recipients, the highest risk is having a new CMV exposure (or primary infection), as seen when a CMV-seronegative
Correspondence: Raymund R Razonable Division of Infectious Diseases, Mayo Clinic, 200 First Street SW, Rochester, MN, USA

Tel +I 5072843747

Email Razonable.raymund@mayo.edu 
Table I CMV risk factors for solid organ transplant recipients

\begin{tabular}{|c|c|c|c|}
\hline Serostatus & Mild risk & $\begin{array}{l}\text { Moderate } \\
\text { risk }\end{array}$ & $\begin{array}{l}\text { High } \\
\text { risk }\end{array}$ \\
\hline $\begin{array}{l}D+/ R- \\
D+/ R+ \\
D-/ R+ \\
D-/ R-\end{array}$ & $\begin{array}{l}X \text { (from pri- } \\
\text { mary infec- } \\
\text { tion acquired } \\
\text { in the } \\
\text { community) }\end{array}$ & $\begin{array}{l}x x \\
x\end{array}$ & $x X$ \\
\hline $\begin{array}{l}\text { Transplanted organ } \\
\text { Lung } \\
\text { Pancreas } \\
\text { Intestinal } \\
\text { Heart } \\
\text { Kidney } \\
\text { Liver } \\
\text { Induction } \\
\text { immunotherapy } \\
\text { Alemtuzumab } \\
\text { ATG } \\
\text { Basiliximab }\end{array}$ & & $\begin{array}{l}X \\
X \\
X\end{array}$ & $\begin{array}{l}X X \\
X X \\
x X \\
\\
\\
x x \\
x x\end{array}$ \\
\hline $\begin{array}{l}\text { Maintenance immu- } \\
\text { nosuppression } \\
\text { Calcineurin inhibitors/ } \\
\text { antimetabolites } \\
\text { Steroids } \\
\text { mTOR inhibitors } \\
\text { Mycophenolate } \\
\text { mophetil }\end{array}$ & $x$ & $\begin{array}{l}X \\
X(\text { standard } \\
\text { dose of }=<2 \\
\text { g/day) }\end{array}$ & $\begin{array}{l}X \\
X \text { (high } \\
\text { dose of } \\
\geq 3 \text { g/day) }\end{array}$ \\
\hline
\end{tabular}

Notes: $X X$ is higher risk than $X$ for the same risk category

Risk factors for CMV in allogeneic hematopoietic stem cell transplant recipients are listed in the text.

Abbreviations: CMV cytomegalovirus; ATG, anti-thymocyte globulin, mTOR, mammalian target of rapamycin.

patient receives an organ allograft that contains latently infected cells from CMV-seropositive donor $(\mathrm{D}+/ \mathrm{R}-){ }^{3}{ }^{3} \mathrm{In}$ addition, the degree of T-cell impairment, as defined by induction immunotherapy (eg, use of T-cell depleting agents), higher doses of maintenance immunosuppression (eg, high-dose steroids or mycophenolate mofetil) and episodes of acute cellular rejection and their treatment ${ }^{4}$ increases the risk and severity of CMV. Finally, certain types of SOTs (eg, lungs, pancreas and intestine) have relatively higher risk compared to kidney recipients. ${ }^{5,6}$

Among allogeneic HSCT recipients, the highest risk are CMV-seropositive recipients, especially if they received stem cells from $\mathrm{CMV}$-seronegative donors (reverse $\mathrm{D}-\mathrm{R}+$ mismatch), those with acute or chronic graft-vs-host disease (GVHD) and require higher doses of steroids and other immunosuppressive drugs, ${ }^{7}$ those with at least one mismatch at one of the specified three human leukocyte antigen (HLA) gene loci, or have a haploidentical donor. In addition, HSCT recipients of naïve (such as umbilical cord blood as the stem-cell source) or ex vivo T-cell-depleted grafts have a very high risk of CMV infection. ${ }^{8}$

\section{Clinical effects of CMV in transplantation}

When symptomatic infection occurs in SOT recipients, it can present as either CMV syndrome or end-organ disease. CMV syndrome (a term that is exclusively used in SOT recipients) is characterized by systemic symptoms (fevers, fatigue), leukopenia, atypical lymphocytosis and thrombocytopenia during CMV replication (as evidenced by detectable CMV DNA in the blood by nucleic acid testing, NAT). End-organ CMV disease is frequently seen in the transplanted allograft, especially among CMV D+/R-SOT recipients, likely due to the reactivation of the latent $\mathrm{CMV}$ in the transplanted organ. ${ }^{4}$ Most commonly, however, the affected organ is the gastrointestinal tract, followed by the lungs, although the virus can invade any other organ system including the liver, retina, and the nervous system.

Among allogeneic HSCT recipients, CMV can present as either viremia (active infection) or less commonly end-organ disease. In the absence of preventive strategy, $70-80 \%$ of allogeneic HSCT recipients develop early CMV reactivation, including a third who develop reactivation in the lungs. ${ }^{9}$ With prophylactic and preemptive antiviral therapies, late-onset CMV infection and disease has emerged as a major problem, especially among patients who are lymphopenic and remain severely immune-deficient, and this remains associated with nonhematologic relapse mortality (p. 125). ${ }^{10}$

In general, CMV infection has been associated with increased morbidity and poor long-term survival in SOT and HSCT recipients. In SOT population, CMV is associated with tissue and organ damage, acute cellular ejection, and increased risk of other opportunistic bacterial and fungal infections. ${ }^{11,12}$ Among the HSCT recipients, CMV has been associated with a higher incidence of GVHD, ${ }^{13,14}$ and increased mortality from infections ${ }^{15,16}$ and nonrelapse mortality. ${ }^{10}$ 


\section{Prevention strategies}

The principles, benefits and disadvantages of the two CMV prevention strategies are presented in Table 2. In general, there is no "one-strategy-fits-all" approach for CMV prevention. Instead, the chosen strategy should be based on the type of transplant, risk profile, and riskbenefit ratio. For example, antiviral prophylaxis is a preferred approach among highest-risk SOT recipients, such as $\mathrm{CMV} \mathrm{D}+/ \mathrm{R}-$, lung, intestinal and composite tissue transplant patients. ${ }^{17}$ However, a preemptive therapy approach is also highly effective among CMV D+/Rkidney and liver recipients. In a recently concluded randomized controlled trial of antiviral prophylaxis versus preemptive therapy in $\mathrm{CMV} \mathrm{D}+\mathrm{R}$ - liver transplant recipients, the rate of CMV disease was significantly lower with preemptive therapy, while there was a higher rate of delayed-onset post-prophylaxis CMV disease among patients who received 3 months of valganciclovir prophylaxis (Clinicaltrials.org \#NCT01552369). Among allogeneic HSCT recipients, the preferred approach is CMV surveillance followed by preemptive therapy of asymptomatic CMV replication. This standard approach of CMV surveillance and preemptive therapy has circumvented the negative effect of CMV infection on overall

Table 2 Comparison of current CMV prevention strategies

\begin{tabular}{|l|l|l|}
\hline Strategy & Prophylaxis & $\begin{array}{l}\text { Preemptive } \\
\text { Therapy }\end{array}$ \\
\hline Principle & $\begin{array}{l}\text { Antivirals administered } \\
\text { to all at-risk patients for } \\
\text { a defined period of time } \\
\text { following } \\
\text { transplantation }\end{array}$ & $\begin{array}{l}\text { CMV DNA test (at least } \\
\text { once weekly) } \\
\text { Antiviral therapy started } \\
\text { when viral load exceeds } \\
\text { a certain threshold }\end{array}$ \\
\hline Advantages & $\begin{array}{l}\text { Effectively prevents } \\
\text { early CMV reactivation }\end{array}$ & $\begin{array}{l}\text { Reduced medication } \\
\text { cost } \\
\text { Lower risk of drug toxi- } \\
\text { city } \\
\text { Allows immune } \\
\text { reconstitution }\end{array}$ \\
\hline Disadvantages & $\begin{array}{l}\text { High rates of medication } \\
\text { side effects } \\
\text { Increased drug cost } \\
\text { Risk for delayed-onset } \\
\text { CMV disease }\end{array}$ & $\begin{array}{l}\text { Does not prevent early } \\
\text { CMV reactivation } \\
\text { Escape CMV infections } \\
\text { (not detected by weekly } \\
\text { CMV NAT) } \\
\text { Burden, logistics and } \\
\text { cost of weekly surveil- } \\
\text { lance labs }\end{array}$ \\
\hline
\end{tabular}

Abbreviations: CMV, Cytomegalovirus; NAT, Nucleic Amplification Test. survival after HSCT (p. 1883). ${ }^{18}$ In contrast, the neutropenic effects of ganciclovir and valganciclovir prophylaxis could delay or impair hematopoietic stem cell engraftment after HSCT, or increase the risk of invasive fungal infections. ${ }^{19}$

\section{Antiviral drugs}

CMV management strategies rely heavily on the use of antiviral drugs that inhibit CMV replication through their effects on CMV DNA polymerase (Table 3). Among them, ganciclovir and valganciclovir are the preferred agents. Because of nephrotoxicity, foscarnet and cidofovir are considered alternative options, and are used mainly for patients who have ganciclovir intolerance or have infections due to ganciclovir resistant virus. There are a few antiviral drugs under clinical investigation such as maribavir (a UL97 kinase inhibitor) and brincidofovir (lipid formulation of cidofovir). Letermovir, a viral terminase inhibitor, is the newest FDA-approved drug for CMV prophylaxis in allogeneic HSCT recipients. In this review, we will discuss the role of letermovir, and its place in CMV management after transplantation.

\section{DNA polymerase inhibitors}

Ganciclovir (intravenous) and valganciclovir are the backbone and first-line antivirals for prevention and treatment of CMV disease after transplantation. Valganciclovir is the oral formulation that gets hydrolyzed and transformed into ganciclovir in the intestinal tract and it virtually exists only in the form of ganciclovir in the systemic circulation. It is highly bioavailable and systemic drug exposure is similar to intravenous ganciclovir. ${ }^{4}$ Ganciclovir is a $2^{\prime}$-deoxyguanosine analogue that acts as a competitive substrate for CMV DNA synthesis, which is catalyzed by UL54-encoded polymerase.$^{20}$ For it to act as a competitive substrate, ganciclovir needs to be activated through the process of phosphorylation, a reaction that is catalyzed initially by CMV UL97-encoded kinase and subsequently by human kinases. Ganciclovir-triphosphate serves as competitive substrate for CMV DNA synthesis, and its incorporation effectively halts CMV DNA synthesis. Genetic mutations in UL97 and UL54 may confer resistance to ganciclovir, as a result of impaired drug activation (phosphorylation) or binding (polymerase), respectively. Ganciclovir is excreted in the kidneys, and dosage should be adjusted based on renal function. ${ }^{21}$ Myelosuppression, most 
Table 3 Characteristics of antiviral drugs approved for cytomegalovirus

\begin{tabular}{|c|c|c|c|c|}
\hline $\begin{array}{l}\text { Drug } \\
\text { Name }\end{array}$ & $\begin{array}{l}\text { Ganciclovir and } \\
\text { valganciclovir }\end{array}$ & Foscarnet & Cidofovir & Letermovir \\
\hline $\begin{array}{l}\text { Mechanism } \\
\text { of action }\end{array}$ & $\begin{array}{l}\text { 2'-deoxyguanosine analogue } \\
\text { Competitive binding to UL54 } \\
\text { DNA polymerase } \\
\text { Needs phosphorylation by CMV } \\
\text { (UL97 encoded) and host } \\
\text { kinases } \\
\text { Virostatic agent }\end{array}$ & $\begin{array}{l}\text { Pyrophosphate analogue } \\
\text { Noncompetitive inhibitor of many } \\
\text { RNA and DNA polymerases } \\
\text { (UL54 DNA polymerase in CMV) } \\
\text { Virostatic agent }\end{array}$ & $\begin{array}{l}\text { Acyclic monophosphate deoxycyti- } \\
\text { dine analogue } \\
\text { Competitive substrate of UL54 DNA } \\
\text { polymerase leads to inhibition of viral } \\
\text { DNA synthesis through incorpora- } \\
\text { tion into growing viral DNA chain } \\
\text { Virostatic agent }\end{array}$ & $\begin{array}{l}\text { Inhibits viral ter- } \\
\text { minase complex, } \\
\text { encoded by genes } \\
\text { UL56, UL5 I and } \\
\text { UL89 } \\
\text { Virostatic agent }\end{array}$ \\
\hline $\begin{array}{l}\text { Indications/ } \\
\text { uses }\end{array}$ & $\begin{array}{l}\text { CMV retinitis } \\
\text { CMV prophylaxis SOT } \\
\text { Non-FDA uses: } \\
\text { CMV disease } \\
\text { CMV preemptive strategy } \\
\text { CMV prophylaxis in HSCT }\end{array}$ & $\begin{array}{l}\text { CMV retinitis } \\
\text { Non-FDA uses: } \\
\text { second line for GCV resistant } \\
\text { CMV disease therapy, prophylaxis } \\
\text { or preemptive therapy }\end{array}$ & $\begin{array}{l}\text { CMV retinitis } \\
\text { Non-FDA uses: } \\
\text { 2nd line for GCV resistant CMV } \\
\text { disease therapy, prophylaxis or pre- } \\
\text { emptive therapy }\end{array}$ & $\begin{array}{l}\text { CMV prophylaxis } \\
\text { in CMV- } \\
\text { seropositive } \\
\text { HSCT recipients }\end{array}$ \\
\hline Formulations & $\begin{array}{l}\text { GCV IV only } \\
\text { VGCV oral }\end{array}$ & IV only & $\begin{array}{l}\text { IV only } \\
\text { Lipid conjugate not yet approved } \\
\text { (brincidofovir) }\end{array}$ & IV and PO \\
\hline $\begin{array}{l}\text { Adverse } \\
\text { effects }\end{array}$ & $\begin{array}{l}\text { Pancytopenia and myelosup- } \\
\text { pression (leukopenia/neutrope- } \\
\text { nia++) } \\
\text { Renal injury } \\
\text { Diarrhea } \\
\text { Less common: pruritus, nausea, } \\
\text { fever, torsade de pointe }\end{array}$ & $\begin{array}{l}\text { Renal injury } \\
\text { Electrolytes wasting } \\
\text { Neutropenia } \\
\text { Less common: headache, diarrhea, } \\
\text { fever, QTc prolongation }\end{array}$ & $\begin{array}{l}\text { Renal injury } \\
\text { Proteinuria } \\
\text { Neutropenia } \\
\text { Ocular toxicity (iritis, uveitis, } \\
\text { amblyopia) } \\
\text { Less common: headaches, shivering, } \\
\text { rash, alopecia, dyspnea }\end{array}$ & $\begin{array}{l}\text { Uncommon, } \\
\text { mainly Gl (gastri- } \\
\text { tis, nausea), dys- } \\
\text { pnea, hepatitis }\end{array}$ \\
\hline $\begin{array}{l}\text { Resistance } \\
\text { mechanism }\end{array}$ & $\begin{array}{l}\text { Mutations in UL97 gene prevent } \\
\text { activation of drug } \\
\text { Mutations in UL54 gene prevent } \\
\text { binding to DNA polymerase (may } \\
\text { confer cross-resistance with all } \\
\text { DNA-polymerase active } \\
\text { antivirals) }\end{array}$ & $\begin{array}{l}\text { Mutations in UL54 gene prevent } \\
\text { binding to DNA polymerase (may } \\
\text { confer cross-resistance with all } \\
\text { DNA-polymerase active antivirals) }\end{array}$ & $\begin{array}{l}\text { Mutations in UL54 gene prevent } \\
\text { binding to DNA polymerase (may } \\
\text { confer cross-resistance with all } \\
\text { DNA-polymerase active antivirals) }\end{array}$ & $\begin{array}{l}\text { Mutations in } \\
\text { UL56 gene } \\
\text { Less commonly, } \\
\text { mutations in } \\
\text { UL5I or UL89 } \\
\text { genes }\end{array}$ \\
\hline
\end{tabular}

Abbreviations: CMV, cytomegalovirus; GVC, Ganciclovir; HSCT, hematopoietic stem cell transplant; IV, intravenous; PO, oral; GI, gastrointestinal; VGCV, Valganciclovir.

commonly neutropenia and leukopenia, is the major adverse effect of ganciclovir.

Foscarnet is a second-line agent for CMV, and it is mainly used for the treatment of ganciclovir-resistant $\mathrm{CMV}$, or when use of ganciclovir is contraindicated. It is only available in intravenous route, highly nephrotoxic and requires close monitoring of serum creatinine and bivalent electrolytes. ${ }^{22}$ Foscarnet is a pyrophosphate analogue that inhibits UL54-encoded CMV DNA polymerase by noncompetitive binding. ${ }^{20}$ Specific mutations in UL54 render CMV DNA polymerase less susceptible to foscarnet binding.
Cidofovir is a nephrotoxic broad-spectrum acyclic monophosphate deoxycytidine analogue that serves as a secondline agent for treatment of ganciclovir-resistant or refractory $\mathrm{CMV}$ and those intolerant to ganciclovir or foscarnet. ${ }^{23}$ It is available in intravenous formulation. An investigational oral lipid formulation (called brincidofovir) is under clinical evaluation. ${ }^{20}$ Cidofovir acts as a competitive substrate for UL54-encoded CMV DNA polymerase, and its incorporation effectively halts CMV DNA synthesis. Specific mutations in UL54 may lead to cidofovir resistance. Often, crossresistance between ganciclovir and cidofovir is observed, while mono-resistance to cidofovir is uncommon. 
The high rates of adverse effects due to the use of CMV DNA polymerase inhibitors, and the emergence of drug-resistant CMV led to major efforts of developing novel antiCMV agents, most notably letermovir.

\section{Letermovir-viral terminase inhibitor}

Letermovir is a 3,4-dihydro-quinazoline-4-yl-acetic acid derivative that inhibits viral terminase complex inhibitor, encoded by $U L 56 .{ }^{24}$ In contrast to CMV DNA polymerase inhibitors that inhibits CMV DNA synthesis, the effect of letermovir is in the prevention of the cleavage of long DNA concatamers into individual viral subunits, thereby resulting in noninfectious long DNA particles. ${ }^{20}$ Letermovir is active solely against human CMV, and it has no activity against the other herpesviruses. ${ }^{25}$ This narrow antiviral effect is important to emphasize clinically since the use of letermovir for CMV prophylaxis should be complemented by use of other antiviral drugs for the prevention of herpes simplex virus (HSV) (eg, acyclovir). Table 4 summarizes the characteristics of letermovir.

Letermovir is a highly bioavailable drug (94\%) in studies performed among healthy individuals. However, this drops to $35 \%$ among allogeneic HSCT recipients; it is not clear whether this is due to impaired absorption (from mucosal inflammation) or due to drug interactions. In allogeneic HSCT recipients, the catabolism of letermovir is reduced (and systemic levels increased) when co-administered with cyclosporine; this interaction appears to be bidirectional since letermovir also increases cyclosporine exposure. The dose of letermovir is therefore reduced among patients who are receiving cyclosporine. However, this bidirectional interaction was not observed with tacrolimus, although tacrolimus doses need to be adjusted when coadministered with letermovir. ${ }^{26}$ Pharmacokinetic studies also highlight the interaction between letermovir and voriconazole (but not posaconazole); letermovir reduces voriconazole exposure, suggesting that letermovir may be a CYP2C9/19 inducer. ${ }^{27}$

Letermovir is available in oral and intravenous formulations. Dose adjustments are not needed for patients with creatinine clearances $\geq 10 \mathrm{~mL} /$ minute, and it is not affected by hemodialysis. ${ }^{28}$ Intra and interindividual variability is minimal, and standard doses result in constant serum trough levels above EC $_{90}$ values. $^{29}$
Table 4 Letermovir key characteristics

\begin{tabular}{|c|c|}
\hline Molecule & $\begin{array}{l}\text { 3,4-dihydro-quinazoline-4-yl-acetic acid } \\
\text { derivative }\end{array}$ \\
\hline Mechanism of action & Inhibits terminase complex subunit pUL56 \\
\hline Spectrum of activity & $\begin{array}{l}\text { Only active against CMV (no activity } \\
\text { against HSV) }\end{array}$ \\
\hline Bioavailability & $\begin{array}{l}94 \% \text { healthy individuals } \\
35 \% \text { in HSCT (increased to } 85 \% \text { with } \\
\text { cyclosporine) }\end{array}$ \\
\hline Excretion & $93 \%$ in feces, mostly as unchanged drug \\
\hline Dosing & $\begin{array}{l}480 \mathrm{mg} \text { daily ( } 240 \mathrm{mg} \text { if administered with } \\
\text { cyclosporine) for prophylaxis in HSCT } \\
\text { No dose adjustment for renal dysfunction }\end{array}$ \\
\hline Side effects & $\begin{array}{l}\text { Uncommon, mainly GI (gastritis, nausea), } \\
\text { dyspnea, hepatitis }\end{array}$ \\
\hline Drug interactions & $\begin{array}{l}\text { Reduces exposure to voriconazole } \\
\text { Increases exposure to tacrolimus, cyclos- } \\
\text { porine, midazolam } \\
\text { Letermovir exposure increased with } \\
\text { cyclosporine }\end{array}$ \\
\hline $\begin{array}{l}\text { Current FDA } \\
\text { approved indication }\end{array}$ & $\begin{array}{l}\mathrm{CMV} \text { prophylaxis in CMV-seropositive HSCT } \\
\text { recipients }\end{array}$ \\
\hline Ongoing trials & $\begin{array}{l}\text { Use of letermovir for CMV prophylaxis in } \\
\text { CMV-seronegative kidney transplant recipi- } \\
\text { ents clinicaltrials.gov NCT03443869 }\end{array}$ \\
\hline
\end{tabular}

Abbreviations: CMV, cytomegalovirus; HSV, herpes simplex virus; HSCT, hematopoietic stell cell transplant; $\mathrm{Gl}$, gastrointestinal.

\section{Approved and potential clinical uses of letermovir \\ Letermovir is approved for CMV prophylaxis in allogeneic HSCT recipients}

In November 2017, letermovir was approved by the US FDA for prophylaxis to prevent CMV infection and disease in CMV-seropositive allogeneic HSCT recipients. ${ }^{30}$ With this approval, letermovir brings the strategy of antiviral prophylaxis as a viable option for CMV prevention in HSCT recipients. Prior to its approval, the standard of CMV prevention among allogeneic HSCT recipients was surveillance with preemptive therapy - an effort to avoid the leukopenic and neutropenic effects of ganciclovir and valganciclovir prophylaxis. The lingering question now, and subject to debate, is - will the approval of letermovir for CMV prophylaxis change this current standard of care? ${ }^{31}$

The approval of letermovir for CMV prophylaxis was based on results of a phase III randomized, placebo- 
controlled trial that enrolled $565 \mathrm{CMV}$-seropositive allogeneic HSCT recipients. ${ }^{8}$ In this study, HSCT recipients were randomized $2: 1$ to receive letermovir $480 \mathrm{mg}$ daily (or $240 \mathrm{mg}$ if receiving cyclosporine) or placebo (standard of care; CMV surveillance and preemptive therapy) for 14 weeks after transplantation. The primary endpoint was the proportion of patients with clinically significant CMV infection-defined as the need for preemptive therapy or having CMV disease-between patients on letermovir prophylaxis or placebo (standard of care). Of the 565 patients enrolled, 495 patients did not have CMV replication at baseline and were randomized (325 in letermovir group and 170 in placebo group). By week 24, patients who were randomized to letermovir prophylaxis had significantly lower incidence of clinically significant CMV122 of 325 patients $(37.5 \%)$ in letermovir vs 103 of 170 $(60.6 \%)$ in placebo; $P<0.001$. This significant difference was evident early at week $14-62$ of $325(19.1 \%)$ in letermovir vs 85 of $170(50 \%)$ in placebo; $P<0.001$. It is worth emphasizing that the incidence of clinically significant CMV in the letermovir group increased after week 18 (termed as delayed onset post-prophylaxis CMV infection), postulated to be occurring more among patients with impaired immunity such as those with GVHD and subsequent therapy with glucocorticoids. Nonetheless, the significant reduction in rate of clinically significant CMV infection was observed for the standard-risk and high-risk groups. In the subgroup analysis, patients were considered high risk if they have at least one of the following: (1) related donor with at least one mismatch at one of the specified three HLA gene loci (HLA-A, B, or DR); (2) having an unrelated donor with at least one mismatch at one of the specified four HLA gene loci (HLA-A, B, C, and DRB1); (3) having a haploidentical donor; (4) the use of umbilical cord blood as the stem-cell source; (5) the use of ex vivo T-cell-depleted grafts; and (6) having GVHD of grade 2 or higher that led to the use of $1 \mathrm{mg} / \mathrm{kg} / \mathrm{day}$ or more of prednisone (or its equivalent).

Survival outcome was assessed as a secondary endpoint; there was a trend towards lower all-cause mortality among allogeneic HSCT recipients who received letermovir prophylaxis, but this did not reach statistical significance. All patients in the study received HSV prophylaxis with acyclovir, valacyclovir or famciclovir (since letermovir has no activity against HSV).

The approval of letermovir paves the way for antiviral prophylaxis as another safe and effective option for the prevention of CMV infection and disease in allogeneic
HSCT recipients. However, the low rate of CMV disease $(<5 \%)^{8}$ with current standard approach, where HSCT patients are monitored at least once weekly with CMV NAT and treated aggressively with intravenous ganciclovir or valganciclovir may argue against changing the current standard practice. Moreover, cost consideration is part of this debate; literature on a cost-benefit analysis of letermovir compared to CMV surveillance/preemptive therapy would be useful. The trend for better survival, at least early after transplantation, is an interesting observation that could sway in favor of letermovir prophylaxis, if this is proven to be true. For now, we believe that letermovir prophylaxis and CMV surveillance/preemptive therapy are equally acceptable options for CMV prevention after allogeneic HSCT. However, letermovir prophylaxis should be strongly considered for those considered high-risk for CMV reactivation, such as recipients of umbilical cords and T-cell-depleted grafts (Table 1). If letermovir prophylaxis is chosen as the method for prevention, we emphasize the need to provide antiviral drugs for HSV prophylaxis (as letermovir has no activity against HSV, varicella zoster and other herpesviruses). Weekly CMV NAT is also encouraged if using letermovir for prophylaxis since there is a risk of breakthrough infections (occurring during prophylaxis). Breakthrough CMV infections were reported in the randomized clinical trial, and in several case reports when letermovir was used as prophylaxis. ${ }^{8}$ There was a report describing a 54-year-old woman who received haploidentical cord blood transplantation for acute myeloid leukemia and developed worsening CMV viremia with pneumonia during letermovir prophylaxis. Genetic resistance testing demonstrated UL56 C325F mutation. ${ }^{32}$ It is also important to keep in mind that letermovir had clinically relevant drug-drug interactions (eg, cyclosporine and voriconazole) and dose adjustments are necessary. Finally, delayed-onset postprophylaxis CMV infection and disease is an anticipated outcome, so patients should be monitored clinically and virologically after letermovir prophylaxis is discontinued, in order to diagnose and treat CMV replication early and aggressively.

\section{Role of letermovir for treatment of CMV infection and disease in HSCT recipients}

Letermovir is not approved for preemptive therapy of asymptomatic infection. There is no clinical trial that is planned for this indication. However, in the phase III letermovir prophylaxis trial, ${ }^{8}$ some of the patients had detectable CMV replication at enrollment, with a median CMV viral 
load of 150 copies/mL. These patients were subsequently randomized to letermovir or placebo (standard of care; CMV surveillance and preemptive therapy with ganciclovir or valganciclovir). In a post-hoc analysis, the rate of clinically significant CMV infection at week 14 was significantly lower in letermovir group (15 of 48 patients; $31.3 \%$ ) compared to placebo (17 of 22 patients; $77.3 \%$ ). When missing information was imputed as having met the primary endpoint, the incidence of clinically significant CMV infection was $45.8 \%$ (22 of 48 patients) for letermovir and $90.9 \%$ (20 of 22 patients) for placebo. It was also reported that 6 of 8 patients with quantifiable CMV viral loads one week after starting the clinical trial drug (5 in letermovir group and 1 in placebo group) had subsequently undetectable viral loads. ${ }^{33}$ This data suggests that letermovir may be effective for patients with active CMV replication. However, we strongly emphasize that this indication has not been subjected to controlled clinical trials, and thus, currently, letermovir should not be used for preemptive therapy of asymptomatic CMV reactivation in allogeneic HSCT recipients. Likewise, there are no solid clinical data to support the use of letermovir for treatment of CMV disease after HSCT, even for those with ganciclovirresistant CMV. Despite the recent data presented in IDweek $^{33}$ that may look promising for implementing letermovir as potential therapy, this is not an approved clinical indication. ${ }^{34}$ Currently, intravenous ganciclovir and valganciclovir are the recommended drugs for the preemptive therapy of asymptomatic CMV infection and treatment of CMV disease in allogeneic HSCT recipients.

\section{Role of letermovir for CMV prophylaxis in SOT recipients}

Letermovir is not approved for any clinical indications in SOT recipients. However, it is being investigated for its role as CMV prophylaxis in high-risk CMV D+/R-SOT recipients. In May 2018, a clinical trial started recruiting participants to determine if letermovir prophylaxis is noninferior to the current standard, valganciclovir prophylaxis, for prevention of CMV disease in high-risk CMV D+/Rkidney transplant recipients (clinicaltrials.gov NCT03443869). This phase III study is aiming to enroll 600 kidney transplant recipients, who will be randomized equally in a double blinded manner in two arms: one arm will receive letermovir, acyclovir and placebo and the other arm will receive valganciclovir and two placebos (to letermovir and acyclovir). Antiviral prophylaxis will be carried out for 28 weeks. The primary outcome of the study is CMV disease incidence at 52 weeks. Secondary outcomes include CMV disease at 28 weeks (end of prophylaxis), time of onset of CMV disease up to 52 weeks of follow-up, adverse events occurrences up to 30 weeks and serious drug-related adverse events up to 52 weeks of follow-up. The study will exclude patients with prior exposure to $\mathrm{CMV}$ and will only enroll recipients who are CMV-seronegative receiving a donor CMV-seropositive kidney. Estimated study completion date is September of 2021.

There has been a case report of the use of letermovir as secondary prophylaxis in a patient with ganciclovirresistant $\mathrm{CMV}$ infection that was initially treated with cidofovir and foscarnet. ${ }^{35}$ However, in the absence of randomized controlled trial results to demonstrate its efficacy and safety, letermovir is not yet recommended for antiviral prophylaxis in SOT recipients.

\section{Role of letermovir for therapy of CMV infection and disease in SOT recipients}

Letermovir has been investigated as a potential therapeutic option for preemptive strategy in kidney transplant recipients. The study was a phase IIa randomized, controlled, open-label study that evaluated two doses of letermovir (40 mg twice daily or $80 \mathrm{mg}$ once daily) compared with standard of care (most commonly ganciclovir or valganciclovir) in kidney transplant recipients. ${ }^{29}$ Interventions were initiated according to local practice upon the detection of CMV replication by NAT. All groups (letermovir and standard of care) had significant reduction in CMV DNA by day 14, although the rate of decline was faster in the standard of care group. This study was designed as a proof-of-concept trial with a small sample size, thus not randomizing equally to factors such as immunosuppressant regimen, comedications, and baseline viral loads. Notably, as the study was conducted before letermovir dosing was fully established, a dose of $80 \mathrm{mg}$ per day was used (either once daily or divided in two doses). Letermovir is now approved at the dose of $480 \mathrm{mg}$ daily for CMV prophylaxis in HSCT. Whether this would have had an impact on the study results is unclear, as the authors report consistent trough concentrations above the targeted $\mathrm{EC}_{90}$. It was also noted that, among the patients who developed CMV disease in this cohort, three had resistant strains (UL97 and UL54 mutations conferring resistance to $\mathrm{GCV}$, cidofovir and foscarnet) and responded well to letermovir therapy; this information suggests that it can have potential indications for treatment of drug-resistant CMV. 


\section{Role of letermovir for treatment of drug-resistant CMV infection and disease}

There are few case reports that have emerged to highlight the potential use of letermovir as treatment of CMV disease, including multidrug-resistant $\mathrm{CMV}$ infections. In the first case, a patient developed breakthrough CMV pneumonitis and retinitis while receiving valganciclovir prophylaxis at 5 months after lung transplantation. Sequential or concomittant treatment with intravenous ganciclovir, foscarnet, cidofovir, leflunomide, CMV hyperimmune immunoglobulin, brincidofovir and artemisinin derivative, along with reduction of tacrolimus and cessation of mycophenolate mofetil was not succesful. ${ }^{36}$ Letermovir was used at a $120 \mathrm{mg}$ daily dose for the first 16 days, and then increased to $240 \mathrm{mg}$ daily, which led to virologic and clinical improvement. In a second case, a patient developed postprophylaxis delayed-onset CMV viremia occurring 53 weeks after lung transplant ( 2 weeks after stopping valganciclovir prophylaxis); this patient was initially treated with intravenous ganciclovir and immunosuppressive medication changes (switch from cyclosporine to tacrolimus; initiation of leflunomide and everolimus), then foscarnet when CMV infection recurred with UL97 mutation. When the infection relapsed again, letermovir $(480 \mathrm{mg}$ once daily) was used, leading to gradual decline in viral load for 5 weeks. However, the patient had viral rebound while on letermovir therapy. Viral genetic analysis revealed UL56 mutation C325Y, conferring absolute resistance to letermovir. ${ }^{37}$ Most recently, letermovir was used as salvage therapy in a series of 4 SOT recipients with resistant CMV retinitis, with 3 patients failing treatment. ${ }^{38}$ In two of the three cases that failed letermovir treatment, UL56 mutation in codon 325 was documented to emerge during treatment.

One of the major risk factors for resistant and refractory CMV infection is the severity of T-cell impairment. ${ }^{4}$ Hence, it is important to restore cell-mediated immunity in transplant recipients, particularly CMV-specific T-cell immunity. ${ }^{1,39}$ Combining immunosuppression reduction with letermovir therapy (or any antiviral drug for that matter) may improve CMV control and accelerate viremia clearance, hence reducing prolonged letermovir exposure and emergence of resistance.

Interestingly, there is in vitro synergy that has been suggested between letermovir and DNA polymerase inhibitors (ganciclovir, foscarnet and cidofovir) ${ }^{40}$ as well as the novel investigational CMV UL97 kinase inhibitor maribavir. ${ }^{41}$ Whether combination therapy with letermovir and another antiCMV drug is more efficacious and reduces risk of resistance will have to be investigated in a clinical trial.

\section{Adverse reactions of letermovir}

Letermovir is a generally well tolerated drug. The most common reported adverse events during clinical trials is gastrointestinal toxicity (diarrhea, nausea, vomiting). ${ }^{42}$ Other side effects are fatigue, headache, skin rash and peripheral edema. Letermovir does not appear to have significant renal and hematopoietic adverse effects (ie, there is no nephrotoxicity or myelosuppressive effect). ${ }^{28}$ A case of self-limiting hepatitis believed to be due to letermovir has been reported. ${ }^{43}$

\section{Letermovir resistance}

Resistance of CMV to letermovir has emerged, both in experimental and clinical settings. Mutations conferring letermovir resistance is most commonly mapped to UL56 (specifically at codons 231-369; eg V236M, L241P, R369S). Less commonly, mutations of UL51 and UL89 have been implicated in resistance emergence. These three genes collectively encode for viral terminase complex. ${ }^{28}$

In experimental models, letermovir resistance occurred as early as the third in vitro passage with escalating letermovir concentrations (compared to the fifteenth passage with foscarnet). ${ }^{44}$ These in vitro experimental models suggest that letermovir may possess low genetic barrier to resistance. ${ }^{44-46}$ Such low barrier has been demonstrated in the clinical setting, when UL56 V236M resistant mutant was selected during the clinical trial of letermovir prophylaxis. ${ }^{8,47}$ In one study, resistance to letermovir has been reported as early as 102 days into letermovir exposure. $^{38}$

The occurrence of letermovir resistance is believed to be due to the multiplicity of pathways through which CMV can acquire high-grade resistance. ${ }^{44}$ Fold-increases in resistance are not uniform throughout different mutations. Some mutations confer low level resistance (UL51 P91S and UL89 N320H, D344E or M359I), but may have synergistic additive effect when combined with other UL56 gene mutations, such as observed when UL51 P91S is combined with UL56 S229F, L254F and L257I, with 290 -fold increase in letermovir resistance. ${ }^{46}$ The purported low genetic barrier for letermovir resistance and the risk of breakthrough infections cautions against the use of letermovir during infections associated with high levels of 
viral replication, especially in highly immunocompromised transplant recipients. ${ }^{28,36}$

\section{Conclusion}

Letermovir is a novel viral terminase inhibitor that is currently approved for CMV prophylaxis in CMVseropositive allogeneic HSCT recipients. It is safe and effective in reducing the risk of CMV reactivation, when compared to CMV surveillance and preemptive therapy. Whether letermovir will find wider clinical indications beyond CMV prophylaxis in CMV-seropositive recipients of HSCT remains unknown. There is an ongoing clinical trial comparing letermovir to valganciclovir for prevention of CMV disease in CMV D+/R- kidney transplant recipients (NCT03443869), and the results are eagerly awaited. Letermovir favorable tolerability profile as well as its unique mechanism of antiviral activity is a welcome addition to CMV therapeutics. The latter characteristic will pave the way for potential novel clinical indications, including therapy for polymerase inhibitor-resistant virus. It also paves the way for the potential for combination therapy, in the hope of reducing resistance development. However, one would need controlled clinical trials to assess these clinical indications. A major drawback to letermovir use is the reported low genetic barrier to resistance, which could hinder expansion of letermovir's indications into therapy, and potentially even prophylaxis in high-risk groups. As experience with letermovir use accumulates and more randomized clinical trials are conducted, the true scope of letermovir's use and its place in the CMV management will be further realized.

\section{Author contributions}

Both authors contributed to data analysis, drafting and revising the article, gave final approval of the version to be published, and agree to be accountable for all aspects of the work.

\section{Disclosure}

Raymund Razonable reports grants from Roche, personal fees from Merck, personal fees from Novartis, outside the submitted work. The authors report no other conflicts of interest in this work.

\section{References}

1. Gandhi MK, Khanna R. Human cytomegalovirus: clinical aspects, immune regulation, and emerging treatments. Lancet Infect Dis. 2004;4(12):725-738. doi:10.1016/S1473-3099(04)01202-2
2. Crough T, Khanna R. Immunobiology of human cytomegalovirus: from bench to bedside. Clin Microbiol Rev. 2009;22(1):76-98. doi:10.1128/CMR.00034-08

3. Cope AV, Sabin C, Burroughs A, Rolles K, Griffiths PD, Emery VC. Interrelationships among quantity of human cytomegalovirus (HCMV) DNA in blood, donor-recipient serostatus, and administration of methylprednisolone as risk factors for HCMV disease following liver transplantation. $J$ Infect Dis. 1997;176(6):1484-1490. Available from: http://www.ncbi.nlm.nih.gov/pubmed/9395358.

4. Koval CE. Prevention and treatment of cytomegalovirus infections in solid organ transplant recipients. Infect Dis Clin North Am. 2018;32 (3):581-597. doi:10.1016/j.idc.2018.04.008

5. Timpone JG, Yimen M, Cox S, et al. Resistant cytomegalovirus in intestinal and multivisceral transplant recipients. Transpl Infect Dis. 2016;18(2):202-209. doi:10.1111/tid.12507

6. Timpone JG, Girlanda R, Rudolph L, Fishbein TM. Infections in intestinal and multivisceral transplant recipients. Infect Dis Clin North Am. 2013;27(2):359-377. doi:10.1016/j.idc.2013.02.012

7. Dziedzic M, Sadowska-Krawczenko I, Styczynski J. Risk factors for cytomegalovirus infection after allogeneic hematopoietic cell transplantation in malignancies: proposal for classification. Anticancer Res. 2017;37:12. doi:10.21873/anticanres.12111

8. Marty FM, Ljungman P, Chemaly RF, et al. Letermovir prophylaxis for cytomegalovirus in hematopoietic-cell transplantation. $N$ Engl $J$ Med. 2017:NEJMoa1706640. doi:10.1056/NEJMoa1706640.

9. Gluckman E, Traineau R, Devergie A, Esperou-Bourdeau H, Hirsch I. Prevention and treatment of CMV infection after allogeneic bone marrow transplant. Ann Hematol. 1992;64 Suppl:A158-A61. Available from: http://www.ncbi.nlm.nih.gov/pubmed/1322189.

10. Özdemir E, Saliba RM, Champlin RE, et al. Risk factors associated with late cytomegalovirus reactivation after allogeneic stem cell transplantation for hematological malignancies. Bone Marrow Transplant. 2007;40(2):125-136. doi:10.1038/sj.bmt.1705699

11. Frange P, Leruez-Ville M. Maribavir, brincidofovir et létermovir: nouveaux antiviraux développés pour le traitement des infections à cytomégalovirus. Med Mal Infect. 2018. doi:10.1016/j. medmal.2018.03.006

12. Torre-Cisneros J. Toward the individualization of cytomegalovirus control after solid-organ transplantation: the importance of the "Individual pathogenic balance". Clin Infect Dis. 2009;49 (8):1167-1168. doi:10.1086/605634

13. Broers AE, van Der Holt R, van Esser JW, et al. Increased transplant-related morbidity and mortality in CMV-seropositive patients despite highly effective prevention of CMV disease after allogeneic T-cell-depleted stem cell transplantation. Blood. 2000;95 (7):2240-2245. Available from: http://www.ncbi.nlm.nih.gov/ pubmed/10733491.

14. Cornelissen JJ. Unrelated marrow transplantation for adult patients with poor-risk acute lymphoblastic leukemia: strong graft-versusleukemia effect and risk factors determining outcome. Blood. 2001;97(6):1572-1577. doi:10.1182/blood.V97.6.1572

15. Meijer E, Dekker AW, Rozenberg-Arska M, Weersink AJL, Verdonck LF. Influence of cytomegalovirus seropositivity on outcome after $\mathrm{T}$ cell-depleted bone marrow transplantation: contrasting results between recipients of grafts from related and unrelated donors. Clin Infect Dis. 2002;35(6):703-712. doi:10.1086/342332

16. Craddock C, Szydlo RM, Dazzi F, et al. Cytomegalovirus seropositivity adversely influences outcome after T-depleted unrelated donor transplant in patients with chronic myeloid leukaemia: the case for tailored graft-versus-host disease prophylaxis. $\mathrm{Br} J$ Haematol. 2001;112 (1):228-236. Available from: http://www.ncbi.nlm.nih.gov/pubmed/ 11167809 .

17. Owers DS, Webster AC, Strippoli GF, Kable K, Hodson EM. Preemptive treatment for cytomegalovirus viraemia to prevent cytomegalovirus disease in solid organ transplant recipients. Cochrane Database Syst Rev. 2013. doi:10.1002/14651858.CD005133.pub3 
18. Verduyn Lunel FM, Raymakers R, van Dijk A, van der Wagen L, Minnema MC, Kuball J. Cytomegalovirus status and the outcome of $\mathrm{T}$ cell-replete reduced-intensity allogeneic hematopoietic stem cell transplantation. Biology of Blood and Marrow Transplantation. 2016;22(10):1883-1887. doi: 10.1016/j.bbmt.2016.07.009

19. Boeckh M, Nichols WG. The impact of cytomegalovirus serostatus of donor and recipient before hematopoietic stem cell transplantation in the era of antiviral prophylaxis and preemptive therapy. Blood. 2004;103(6):2003-2008. doi:10.1182/blood-2003-10-3616

20. Razonable RR. Drug-resistant cytomegalovirus. Curr Opin Organ Transplant. 2018;1. doi:10.1097/MOT.0000000000000541

21. Kotton CN, Kumar D, Caliendo AM, et al. The third international consensus guidelines on the management of cytomegalovirus in solid-organ transplantation. Transplantation. 2018;102(6):900-931. doi:10.1097/TP.0000000000002191

22. Avery RK, Arav-Boger R, Marr KA, et al. Outcomes in transplant recipients treated with foscarnet for ganciclovir-resistant or refractory cytomegalovirus infection. Transplantation. 2016;100(10):e74-e80. doi: $10.1097 /$ TP.0000000000001418

23. Razonable RR, Humar A. Cytomegalovirus in solid organ transplantation. Am J Transplant. 2013;13(s4):93-106. doi:10.1111/ ajt.12103

24. Lischka P, Hewlett G, Wunberg $T$, et al. In vitro and in vivo activities of the novel anticytomegalovirus compound AIC246. Antimicrob Agents Chemother. 2010;54(3):1290-1297. doi:10.1128/AAC.01596-09

25. Marschall M, Stamminger T, Urban A, et al. In vitro evaluation of the activities of the novel anticytomegalovirus compound AIC246 (Letermovir) against herpesviruses and other human pathogenic viruses. Antimicrob Agents Chemother. 2012;56(2):1135-1137. doi:10.1128/AAC.05908-11

26. Kropeit D, von Richter O, Stobernack H-P, Rübsamen-Schaeff H, Zimmermann H. Pharmacokinetics and safety of letermovir coadministered with cyclosporine A or tacrolimus in healthy subjects. Clin Pharmacol Drug Dev. 2018;7(1):9-21. doi:10.1002/cpdd.388

27. Marshall WL, McCrea JB, Macha S, et al. Pharmacokinetics and tolerability of letermovir coadministered with azole antifungals (Posaconazole or voriconazole) in healthy subjects. $J$ Clin Pharmacol. 2018;58(7):897-904. doi:10.1002/jcph.1094

28. Razonable RR. Role of letermovir for prevention of cytomegalovirus infection after allogeneic haematopoietic stem cell transplantation. Curr Opin Infect Dis. 2018;31(4):286-291. doi:10.1097/ QCO.0000000000000459

29. Stoelben S, Arns W, Renders L, et al. Preemptive treatment of Cytomegalovirus infection in kidney transplant recipients with letermovir: results of a Phase 2a study. Transpl Int. 2014;27(1):77-86. doi: $10.1111 /$ tri. 12225

30. FDA medical review.; 2018. Available from: https://www.accessdata. fda.gov/drugsatfda_docs/nda/2017/2099390rig1s000, 209940Orig1s000Approv.pdf. Accessed January 13, 2019.

31. Foolad F, Aitken SL, Chemaly RF. Letermovir for the prevention of cytomegalovirus infection in adult cytomegalovirus-seropositive hematopoietic stem cell transplant recipients. Expert Rev Clin Pharmacol. 2018;11(10):931-941. doi:10.1080/ 17512433.2018.1500897

32. Knoll BM, Seiter K, Phillips A, Soave R. Breakthrough cytomegalovirus pneumonia in hematopoietic stem cell transplant recipient on letermovir prophylaxis. Bone Marrow Transplant. 2018. doi:10.1038/ s41409-018-0389-9
33. Marty FM, Ljungman P, Chemaly RF, et al. Outcomes of Patients with Detectable Cytomegalovirus (CMV) DNA at Randomization in the Double-Blind, Placebo-Controlled Phase 3 Trial of Letermovir (LET) Prophylaxis for CMV-Seropositive Allogeneic HematopoieticCell Transplantation (HCT) Recipients. Open Forum Infect Dis. 2018;5(suppl_1):S56-S57. doi:10.1093/ofid/ofy209.136

34. Bowman LJ, Melaragno JI, Brennan DC. Letermovir for the management of cytomegalovirus infection. Expert Opin Investig Drugs. 2017;26(2):235-241. doi:10.1080/13543784.2017.1274733

35. Chong PP, Teiber D, Prokesch BC, et al. Letermovir successfully used for secondary prophylaxis in a heart transplant recipient with ganciclovir-resistant cytomegalovirus syndrome (UL97 mutation). Transpl Infect Dis. 2018;20(5):e12965. doi:10.1111/tid.12965

36. Kaul DR, Stoelben S, Cober E, et al. First report of successful treatment of multidrug-resistant cytomegalovirus disease with the novel anti-CMV compound AIC246. Am J Transplant. 2011;11 (5):1079-1084. doi:10.1111/j.1600-6143.2011.03530.x

37. Cherrier L, Nasar A, Goodlet KJ, Nailor MD, Tokman S, Chou S. Emergence of letermovir resistance in a lung transplant recipient with ganciclovir-resistant cytomegalovirus infection. Am $J$ Transplant. 2018. doi:10.1111/ajt.15135

38. Turner N, Strand A, Grewal DS, et al. Use of letermovir as salvage therapy for drug-resistant cytomegalovirus retinitis. Antimicrob Agents Chemother. 2019;63:3. doi:10.1128/AAC.02337-18

39. Li CR, Greenberg PD, Gilbert MJ, Goodrich JM, Riddell SR. Recovery of HLA-restricted cytomegalovirus (CMV)-specific T-cell responses after allogeneic bone marrow transplant: correlation with CMV disease and effect of ganciclovir prophylaxis. Blood. 1994;83 (7):1971-1979. Available from: http://www.ncbi.nlm.nih.gov/ pubmed $/ 8142663$.

40. Wildum S, Zimmermann H, Lischka $\mathrm{P}$. In vitro drug combination studies of letermovir (AIC246, MK-8228) with approved anti-human cytomegalovirus (HCMV) and anti-HIV compounds in inhibition of HCMV and HIV replication. Antimicrob Agents Chemother. 2015;59 (6):3140-3148. doi:10.1128/AAC.00114-15

41. Chou S, Ercolani RJ, Derakhchan K. Antiviral activity of maribavir in combination with other drugs active against human cytomegalovirus. Antiviral Res. 2018;157:128-133. doi:10.1016/j. antiviral.2018.07.013

42. Chemaly RF, Ullmann AJ, Stoelben S, et al. Letermovir for cytomegalovirus prophylaxis in hematopoietic-cell transplantation. $N$ Engl J Med. 2014;370(19):1781-1789. doi:10.1056/NEJMoa1309533

43. Geswein L. Letermovir associated hepatic transaminitis: A case report. J Oncol Pharm Pract. 2018;107815521878324. doi:10.1177/ 1078155218783245

44. Chou S. Rapid in vitro evolution of human cytomegalovirus UL56 mutations that confer letermovir resistance. Antimicrob Agents Chemother. 2015;59(10):6588-6593. doi:10.1128/AAC.01623-15

45. Chou S. Comparison of cytomegalovirus terminase gene mutations selected after exposure to three distinct inhibitor compounds. Antimicrob Agents Chemother. 2017;61:11. doi:10.1128/ AAC.01325-17

46. Chou S. A third component of the human cytomegalovirus terminase complex is involved in letermovir resistance. Antiviral Res. 2017;148:1-4. doi:10.1016/j.antiviral.2017.10.019

47. Lischka P, Michel D, Zimmermann H. Characterization of cytomegalovirus breakthrough events in a phase 2 prophylaxis trial of letermovir (AIC246, MK 8228). J Infect Dis. 2016;213(1):23-30. doi:10.1093/infdis/jiv352 


\section{Publish your work in this journal}

Infection and Drug Resistance is an international, peer-reviewed openaccess journal that focuses on the optimal treatment of infection (bacterial, fungal and viral) and the development and institution of preventive strategies to minimize the development and spread of resistance. The journal is specifically concerned with the epidemiology of antibiotic resistance and the mechanisms of resistance development and diffusion in both hospitals and the community. The manuscript management system is completely online and includes a very quick and fair peerreview system, which is all easy to use. Visit http://www.dovepress.com/ testimonials.php to read real quotes from published authors.

Submit your manuscript here: https://www.dovepress.com/infection-and-drug-resistance-journal 